\title{
EDITORIAL
}

\section{Portable monitoring in sleep apnoea: the way forward?}

\author{
W.T. McNicholas*, ${ }^{*}$ and P. Lévy ${ }^{\uparrow,+}$
}

D opulation-based epidemiological studies estimate the prevalence of obstructive sleep apnoea syndrome (OSAS) to be at least $2 \%$ of adult females and $4 \%$ of adult males in the developed world based on an apnoea/ hypopnoea frequency (AHI) of $>5$ events $\cdot h^{-1}$ associated with excessive daytime sleepiness. However, a substantial proportion of these individuals are undiagnosed [1-3]. Furthermore, it is very likely that these epidemiological data, now dating back many years, underestimate the current prevalence of OSAS given the dramatic increase in obesity over recent decades [4]. Existing evidence points to OSAS as an independent risk factor for motor vehicle accidents, neurocognitive deficits, and cardiovascular morbidity and mortality [5-8]. Substantial evidence also supports the argument that appropriate treatment of OSAS reduces the risk of these consequences [9-11]. Since the diagnosis of OSAS requires both clinical assessment and objective monitoring of sleep-disordered breathing [12], there are major resource implications for the management of patients with suspected sleep apnoea. However, the perceived need in many healthcare systems to perform costly and labour-intensive polysomnography (PSG) in a sleep laboratory limits patient access to diagnosis and treatment [13]. Commercially available and relatively inexpensive portable monitors might facilitate earlier recognition of disease and faster initiation of treatment, thereby reducing the healthcare burden associated with OSAS [14]. Interest in the clinical application of portable monitoring devices is growing rapidly, and is being used as a mainstay approach to the management of OSAS in some settings. This interest is also supported by the lack of obvious clinical benefit when using full PSG rather than simplified monitoring, as evidenced in a US health technology assessment [15].

\section{WORKSHOP ON RESEARCH PRIORITIES IN THE AMBULATORY MANAGEMENT OF ADULTS WITH OSAS}

An international workshop to determine the research priorities in ambulatory management of adults with OSAS was held on October 15-16, 2007, in Arlington, VA, USA. The overall goal was to promote further research on portable monitor testing, in order to generate the high-quality, empirical evidence needed to determine the role of portable monitors in the ambulatory management of patients with OSAS. The American Thoracic

\footnotetext{
*Respiratory Sleep Disorders Unit, St. Vincent's University Hospital, and ${ }^{\#}$ Conway Institute of Biomolecular and Biomedical Research, University College Dublin, Dublin, Ireland. "INSERM Unit 1042, HP2 laboratory, Joseph Fourier University, and +'Sleep Laboratory, EFCR, Rehabilitation and Physiology Dept, CHU Grenoble, Grenoble, France.

CORRESPONDENCE: W.T. McNicholas, Respiratory Sleep Disorders Unit, St. Vincent's University Hospital, Elm Park, Dublin 4, Ireland. E-mail: walter.mcnicholas@ucd.ie
}

Society (ATS), the American Academy of Sleep Medicine (AASM), the American College of Chest Physicians (ACCP), and the European Respiratory Society (ERS) sponsored the workshop; no funding was received from industry. The objectives of the workshop were: to identify the barriers preventing incorporation of portable monitor testing into clinical management pathways for the evaluation of patients with suspected sleep apnoea; and to develop recommendations regarding research study designs and methodology that will provide information about the potential clinical application of portable monitor testing for patients with the disorder. The present authors represented ERS in the Steering Committee and the proceedings of this workshop have recently been published in the Proceedings of the American Thoracic Society [16].

\section{Current status of portable monitoring}

Despite the intuitive appeal of portable monitor testing, there is limited high-quality empiric evidence concerning their role in the clinical management of patients with sleep apnoea. Inlaboratory PSG, a recording of physiological signals to assess sleep stage and respiration during sleep, remains the gold standard for diagnosis of OSAS and initiation of continuous positive airway pressure (CPAP) treatment, even though the level of evidence is also relatively limited [17]. However, this testing is costly, utilises substantial resources, and requires the supervision of a technologist. Given the high prevalence of sleep apnoea, these considerations provide justification for greater use of portable monitor testing in at least a proportion of patients. Portable monitor testing may have a particularly important role in regions where facilities for laboratory-based management are limited. Indeed, portable monitor testing is increasingly used by healthcare providers, particularly those working in paymentlimited and public healthcare systems $[18,19]$.

Review of recent evidence highlights the need for a focus on outcomes-based studies involving ambulatory monitors. It is important to assess the performance characteristics of specific monitors in the intended clinical context and patient population, and studies should not simply assess the ability of the monitor to reproduce a PSG-derived AHI. Instead, it is imperative to ascertain the clinical outcomes of OSAS management pathways that include the use of portable monitor testing. Economic analyses regarding cost-effectiveness of portable monitoring in the management of sleep apnoea should evaluate health-resource utilisation for the entire clinical management pathway, from diagnosis to treatment outcomes.

Debate continues as to whether portable monitor testing should be used diagnostically in the general population or in 
a subgroup, such as in the diagnosis of patients with a high pre-test likelihood of the disorder. In most current clinical applications, type 3 portable monitors (monitors that record respiratory-related signals but do not record PSG signals for sleep staging) are used for unattended home recordings in the diagnosis of OSAS. Current recommendations are that patients with a negative type 3 recording have an in-laboratory PSG to exclude the possibility of a false-negative study [17]. According to these recommendations, the use of portable monitors to include and exclude the diagnosis of OSAS in the general population would result in a considerable proportion of negative studies, thereby increasing the demand for inlaboratory PSG. However, many centres, particularly those outside North America, accept a negative type 3 study as sufficient if there is a relatively low clinical index of suspicion for OSAS. Limiting portable monitor testing to patients with a high-likelihood of OSAS would minimise the number of negative studies, and thus the need for in-laboratory PSG where diagnosis remains in doubt. Therefore, accurate identification of patients with a high pre-test likelihood of OSAS is important. Clinical prediction rules, including the Multivariable Apnoea Prediction Index, the Sleep Apnoea Clinical Score and the Berlin Questionnaire, have been used for this purpose, but use of these instruments has largely been confined to research studies and they have not been adequately tested in clinical management pathways [20, 21].

There are few published studies assessing portable monitors in specific populations, including the elderly and individuals with other cardiorespiratory and neurological diseases, such as chronic obstructive pulmonary disease, asthma, heart failure and neuromuscular disorders. The initial question that needs to be addressed is whether portable monitor testing is feasible and suitable for the screening and diagnosis of sleep disorders in these specific patient populations. Equally important are the advantages and limitations of these devices compared with conventional assessment methods using attended, in-laboratory PSG, and the difference between the sleep laboratory and home environment.

The current lack of standardisation of commercially available monitors is a major barrier to the incorporation of portable monitor testing into routine clinical management pathways. Although portable monitors for sleep testing are intended primarily for unattended home recordings, they can be used under either attended or unattended conditions and in a variety of locations, including the sleep laboratory and healthcare facilities. The monitors differ widely in the number and type of signals recorded, the sensors used to record the signals, and the electronic processing of the signals. Scoring of the recordings may be totally automated or manual with the assistance of computer software. This lack of uniformity limits the ability to perform meta-analyses and evidence-based reviews, and complicates the ability to compare results across monitors and generalise results obtained with a particular monitor. While further standardisation of portable monitors is needed, important technological questions remain to be answered before we can determine the ideal portable monitor for diagnosis of OSAS. We still need to determine which signals are essential and how the signals should be acquired in terms of sensors employed, sampling rate, and filtering.
Differences in equipment and testing environments, intra-scorer reliability, and the known night-to-night variability in AHI may explain why direct comparisons of results from portable monitor testing and PSG are not closely correlated. Most portable monitors capable of widespread application do not include signals that detect whether the patient is awake or asleep during the recording. The severity of sleep-disordered breathing on these recordings is therefore quantified as the AHI per hour of recording, instead of the number per hour of sleep. In patients with delayed sleep onset and low sleep efficiency, the resulting AHI will underestimate the "true" AHI. Recognising the limitations of studies that directly compare sleep test results between portable monitors and in-laboratory PSG, investigators are starting to perform studies that compare participants randomised to these different pathways in terms of adherence to CPAP treatment, improvements in quality of life, and improvements in other clinical outcomes.

The use of unattended, home portable monitor testing to diagnose patients with OSAS will only alleviate the growing demand for in-laboratory testing if patients can be initiated on CPAP treatment without requiring PSG to establish the optimal CPAP setting. Auto-CPAP units have been successfully used to titrate the fixed pressure setting needed for CPAP treatment in attended and unattended settings [22, 23]. Increasingly, providers are using auto-CPAP instead of CPAP for regular treatment. However, no consensus exists regarding the optimal role of auto-CPAP machines in the clinical management of patients with OSAS.

Ambulatory monitoring should be viewed as a complementary rather than a competitive technology to in-laboratory PSG. To ensure that in-laboratory PSG can be performed as necessary once portable monitoring is incorporated into a diseasemanagement pathway, criteria for this should be specified in the management pathway. There should be clinical guidelines for choosing a portable or an in-laboratory study as the first test, as well as clear definitions for failure of portable monitoring requiring subsequent in-laboratory PSG.

\section{Developments in portable monitoring of sleep and breathing disorders}

In addition to the standard PSG techniques that have been adopted for portable monitor testing, novel technologies have been developed to enhance their performance and application. For example, actigraphy has been evaluated as a surrogate marker of sleep and wakefulness to improve the calculation of AHI [24], and a recently developed non-contact device based on electromagnetic signals shows promise as a surrogate marker of sleep quality [25], and may also be useful in the evaluation of sleep-disordered breathing [26]. Some monitors incorporate other novel sensors that detect cardiac and autonomic responses to sleep apnoea. One such device measures peripheral artery tone from a sensor on the finger that estimates changes in vascular flow, a measure that reflects variations in breathing and sleep-related arousals [27], and may also be used to identify rapid eye movement sleep [28]. Unfortunately, the technological advances in portable monitors far outstrip our knowledge about their utility in clinical testing.

An ideal portable monitor should provide sufficient accuracy for case finding and should be adaptable to self-application, 
comfortable for all-night use and durable enough to withstand nightly transport to and application in the home by inexperienced patients. Moreover, the monitors should provide full disclosure of high-quality primary signals for manual review, and either manual scoring or manual editing of automated scoring. In addition, the device should provide computational algorithms allowing it to be used in a large number of patients. In other words, an ideal portable monitor should contain highquality primary signals without excess labour and perhaps redundancy of signals to provide complementary data acquisition and minimise data loss. The ideal sensors should be easy to apply and provide a core group of reliable signals that include some or all of: oximetry, flow using nasal pressure, electrocardiograph, and/or respiratory effort. The output of sensors should also be accurate, reliable and reproducible.

Pressure for alternative approaches to the current recommended in-laboratory management of patients with OSAS will continue to increase given the cost of PSG, the limited number of laboratory facilities, and the growing clinical demand for more rapid access to testing, which increases with every new study documenting that treatment of sleep apnoea improves functional and cardiovascular outcomes. In this setting, there is growing demand for accurate and reliable portable monitors, and further standardisation of portable monitors is required to allow comparison of study results across monitors. More prospective, high-quality clinical trials are needed to compare home versus in-laboratory testing in terms of treatment outcomes in diverse patient populations. Cost-effectiveness protocols should be routinely incorporated into these clinical trials to collect the data that will allow development of decision analysis models that are based on facts, not assumptions. Current portable monitor technology seems to be most applicable in priority populations such as patients identified as having a high likelihood of sleep apnoea using clinical prediction rules, patients undergoing pre-operative evaluation for bariatric surgery, and obese adults with type 2 diabetes mellitus. Alternative approaches should also be made available to underserved and remote populations that do not have access to so-called "gold standard" testing.

\section{STATEMENT OF INTEREST}

None declared.

\section{REFERENCES}

1 Young T, Palta M, Dempsey J, et al. The occurrence of sleepdisordered breathing among middle-aged adults. N Engl J Med 1993; 328: 1230-1235.

2 Kapur V, Strohl K, Redline S, et al. Underdiagnosis of sleep apnea syndrome in US communities. Sleep Breath 2002; 6: 49-54.

3 Duran J, Esnaola S, Rubio R, et al. Obstructive sleep apneahypopnea and related clinical features in a population-based sample of subjects aged 30 to $70 \mathrm{yr}$. Am J Respir Crit Care Med 2001; 163: 685-689.

4 Brown WV, Fujioka K, Wilson PWF, et al. Obesity: why be concerned? Am J Med 2009; 122: Suppl. 1, S4-S11.

5 Bradley TD, Floras JS. Obstructive sleep apnoea and its cardiovascular consequences. Lancet 2009; 373: 82-93.

6 McNicholas WT, Bonsignore MR. Sleep apnoea as an independent risk factor for cardiovascular disease: current evidence, basic mechanisms and research priorities. Eur Respir J 2007; 29: 156-178.
7 Engleman HM, Douglas NJ. Sleep 4: sleepiness, cognitive function, and quality of life in obstructive sleep apnoea/hypopnoea syndrome. Thorax 2004; 59: 618-622.

8 Punjabi NM, Caffo BS, Goodwin JL, et al. Sleep-disordered breathing and mortality: a prospective cohort study. PLoS Med 2009; 6: e1000132.

9 George CF. Reduction in motor vehicle collisions following treatment of sleep apnoea with nasal CPAP. Thorax 2001; 56: 508-512.

10 Marin JM, Carrizo SJ, Vicente E, et al. Long-term cardiovascular outcomes in men with obstructive sleep apnoea-hypopnoea with or without treatment with continuous positive airway pressure: an observational study. Lancet 2005; 365: 1046-1053.

11 Doherty L, Kiely J, Swan V, et al. Long-term effects of nasal continuous positive airway pressure therapy on cardiovascular outcomes in sleep apnea syndrome. Chest 2005; 127: 2076-2084.

12 McNicholas WT. Diagnosis of obstructive sleep apnea in adults. Proc Am Thorac Soc 2008; 5: 154-160.

13 Flemons WW, Douglas NJ, Kuna ST, et al. Access to diagnosis and treatment of patients with suspected sleep apnea. Am J Respir Crit Care Med 2004; 169: 668-672.

14 Collop NA, Shepard JA, Strollo PJ. Executive summary on the systematic review and practice parameters for portable monitoring in the investigation of suspected sleep apnea in adults. Am J Respir Crit Care Med 2004; 169: 1160-1163.

15 Department of Health and Human Services USA. Home diagnosis of obstructive sleep apnea-hypopnea syndrome. www.cms. hhs.gov/determinationprocess/downloads/id48TA.pdf Date last updated: August 8, 2007. Date last accessed: January, 21: 2011.

16 Kuna ST, Badr MS, Kimoff RJ, et al. An official ATS/AASM/ ACCP/ERS Workshop: research priorities in ambulatory management of adults with obstructive sleep apnea. Proc Am Thorac Soc 2011; 8: 1-16.

17 Kushida CA, Littner MR, Morgenthaler $\mathrm{T}$, et al. Practice parameters for the indications for polysomnography and related procedures: an update for 2005. Sleep 2005; 28: 499-521.

18 Mulgrew AT, Fox N, Ayas NT, et al. Diagnosis and initial management of obstructive sleep apnea without polysomnography. Ann Intern Med 2007; 146: 157-166.

19 Flemons WW, Douglas NJ, Kuna ST, et al. Access to diagnosis and treatment of patients with suspected sleep apnea. Am J Respir Crit Care Med 2004; 169: 668-672.

20 Flemons WW, Reimer MA. Development of a disease-specific health-related quality of life questionnaire for sleep apnea. Am J Respir Crit Care Med 1998; 158: 494-503.

21 Netzer NC, Stoohs RA, Netzer CM, et al. Using the Berlin Questionnaire to identify patients at risk for the sleep apnea syndrome. Ann Intern Med 1999; 131: 485-491.

22 Masa JF, Jimenez A, Duran J, et al. Alternative methods of titrating continuous positive airway pressure: a large multicenter study. Am J Respir Crit Care Med 2004; 170: 1218-1224.

23 Antic NA, Buchan C, Esterman A, et al. A randomized controlled trial of nurse-led care for symptomatic moderate-severe obstructive sleep apnea. Am J Respir Crit Care Med 2009; 179: 501-508.

24 Elbaz M, Roue GM, Lofaso F, et al. Utility of actigraphy in the diagnosis of obstructive sleep apnea. Sleep 2002; 25: 527-531.

25 de Chazal P, Fox N, O'Hare E, et al. Sleep/wake measurement using a non-contact biomotion sensor. J Sleep Res 2010; [Epub ahead of print PMID: 20704645].

26 Zaffaroni A, de Chazal P, Heneghan C, et al. SleepMinder: an innovative contact-free device for the estimation of the apnoeahypopnoea index. Conf Proc IEEE Eng Med Biol Soc 2009; 2009: 7091-7094.

27 Zou D, Grote L, Peker Y, et al. Validation a portable monitoring device for sleep apnea diagnosis in a population based cohort using synchronized home polysomnography. Sleep 2006; 29: 367-374.

28 Lavie P, Schnall RP, Sheffy J, et al. Peripheral vasoconstriction during REM sleep detected by a new plethysmographic method. Nat Med 2000; 6: 606. 